\title{
Implantable vaccines: a solution for immune system manipulation to any antigenic stimulus
}

loanna Zerva, Vasileia Pateraki, Irene Athanassakis*

Department of Biology, University of Crete, Vassilika Vouton, Heraklion 70013, Crete, Greece

\section{Article Info}

\section{Article Notes}

Received: August 29, 2020

Accepted: October 12, 2020

\section{${ }^{*}$ Correspondence:}

Dr. Irene Athanassakis, Department of Biology, University of Crete, Vassilika Vouton, Heraklion 70013, Crete, Greece; Telephone No: +302810394355; Email: athanire@uoc.gr

(C) 2020 Athanassakis I. This article is distributed under the terms of the Creative Commons Attribution 4.0 International License.

\section{Keywords:}

vaccines, antigenic stimulus

antigen delivery

immune system manipulation

immunotherapy, adjuvants

\section{ABSTRACT}

Effective and side-effect-free vaccines are still difficult tasks to achieve for a great majority of antigenic stimuli. Pathogen manipulation to abort infectivity and antigen delivery to ensure immune responsiveness are the major components vaccine technology tries to resolve. However, the development of an immune response is still a complicated matter, lies on hundreds of parameters and any effort towards activation can easily lead to adverse effects, making immunotherapy very difficult to control. The present review attempts to highlight the major parameters affecting immune responsiveness and show that vaccine technology, except from pathogen manipulation and the development of antigen delivery systems, requires attention to additional check-points. Analyzing the recently described personalized implantable vaccine technology, it becomes obvious that the nature of each antigenic stimulus dictates different responsiveness to the organism, which discourages the use of universal adjuvant and antigen-delivery systems. On the contrary, the ex vivo tuning of the immune response proposed by the implantable vaccine technology, allows controllable amendment of the response. The development of personalized technologies is expected to provide valuable tools for the management of human pathology.

\section{Introduction}

The immune system is meant to function in response to any antigenic stimulus, which includes any substance, from small molecules (haptens) to big proteins, nucleic acids, lipids etc. Most of the time these antigens belong to the organism itself (self antigens), but they can also invade the body during various pathogenic infections. The so far acquired knowledge on immune system function has allowed understanding many of the evoked mechanisms, but we are still missing a huge amount of pieces from the puzzle. Thus, the system responds to self antigens by inducing tolerance (suppression of responsiveness) and to non-self antigens by inducing immunity (stimulation of responsiveness). A fine line separates these two very important but different modes of action of the immune system, which once broken will lead either to autoimmunity (response to self antigens) or failure to pathogen destruction (unresponsiveness to pathogen). It has to be noted that cancer, comprising self and nonself antigens, lies between these two modes of action of the immune system delivering controversial signals, which depending on the genetic background and the patho-physiology of the individual may succeed or fail to destroy malignant cells.

Acquired immune defense in vertebrates is represented by a series of organs, cells and mechanisms developed during evolution to recognize and respond to antigens, ensuring species survival 
and propagation. The cognate immune system generally responds to antigenic stimuli through the humoral and cellular pathways, which lead to antibody production and target cell killing, respectively. Antigen recognition occurs through the antigen presentation process, which results in the formation of thermodynamically stable complexes between the major histocompatibility complex (MHC) class I, class II or CD1b proteins with the antigenic epitopes, recognized by helper CD4-positive $T$ cells $\left(\mathrm{T}_{\mathrm{H}}\right)$, cytotoxic CD8-positive $\mathrm{T}$ cells (Tc) or Cd1-restricted $\mathrm{T}$ cells, respectively ${ }^{1-3}$. Such recognition leads to a variety of specific and non-specific responses, which directly or indirectly interfere with the physiology as well as pathophysiology of the organism. Therefore, the initial step for immune response manipulation lies on the successful MHC/antigen complex formation.

In the context of vaccine development, the manipulation of immune response depends on the externally provided technological ability to trigger the organism against the infectious pathogen, or even any kind of antigen, without, however, risking infectivity or mounting severe inflammatory reactions. The present review focuses on the advantages of solid implantable, ex vivo activated scaffolds as to their ability to finely tune the response to an antigenic stimulus over degradable materials delivered to the organism. The parameters discussed herein might sound as basic knowledge of Immunology, but most of the time thoughts do not need to evoke fancy and difficult to achieve technologies.

\section{Antigen presentation process}

In the context of an external antigenic stimulus, the antigen has to be delivered to the organism, up-taken by antigen presenting cells (APCs), degraded and loaded to MHC molecules for interaction and signal delivery to T cells. Each of the above steps is highly regulated and depends on a variety of factors imposing positive or negative regulatory pathways.

\section{Antigen delivery to the organism}

Intrinsic antigens will shut down defensive immune response during the educative process of $\mathrm{T}$ cells in the thymus early in life and the development of peripheral tolerance in adulthood, through various mechanisms that are not within the scope of the present review.

External, environmental antigens are delivered to an organism through contact, inhalation or aspiration and will mount an immune response following the positive and negative regulatory mechanisms, depending on nature of the antigen, its concentration, the delivery site and timing. In most cases, pathogens are encountered by defensive mechanisms for elimination and development of specific memory cells, while antigens within the day-to-day contact of the individual (daily habits) will confront immune tolerance.

Since 1549, when Wan Quan first inoculated smallpox (variolation) to protect against the disease, and the demonstration for the effectiveness of vaccination against smallpox from Jenner in $1796^{4}$, vaccine technology has developed from first to second generation vaccines and research drives towards the use of recombinant DNA techniques with various delivery systems.

First generation vaccines include weakened or killed whole-micro-organisms (Figure 1). Live but attenuated pathogen vaccines, such as smallpox and polio vaccines, are able to induce cytotoxic T-cell responses, helper T-cell responses and antibody-mediated immunity. However, although more effective, there is significant risk of infectivity, where pathogen may revert to a dangerous form able to cause the disease. Killed pathogen vaccines, on the other hand, do not have such risk, but they cannot generate specific Tc responses and may not be effective in eradicating the disease.

The necessity to develop effective with low virulence vaccines led to the second generation vaccines, which are subunit vaccines consisting of defined antigenic peptides or recombinant protein sub-units (Fig. 1). These vaccines are able to generate $T_{H}$ and antibody responses, but not Tc responses, while they require the use of adjuvants for non-specific immune stimulation of the organism, which, however, have non-specific side-effects not effectively monitored nowadays.

Vaccine adjuvants are chemical compounds or macromolecules that augment immune responses of coadministered antigen with minimal toxicity or long lasting immunity on their own. These agents target innate immune responses through two major mechanisms. Adjuvants like aluminum salts, oil-in-water emulsions, and liposomes facilitate antigen depot and thus its uptake by APCs. Other adjuvants like monophosphoryl lipid $\mathrm{A}, \mathrm{CpG}$, or poly $\mathrm{I}: \mathrm{C}$, activate APCs by binding to Toll-like receptors ${ }^{5}$. All these adjuvants cause inflammation at the site of injection but also have a potential for long term side effects and therefore have not been approved for human use. The most widely used adjuvant in the clinics has been aluminumbased mineral salts (alum) ${ }^{6}$, which has a good track record of safety and has been widely used in many licensed vaccines $^{7}$, without, however, lacking disadvantages and side effects ${ }^{8}$. Alternative vaccine adjuvants that are nontoxic, consistently effective, and easy to handle have been hunted for in the past thirty years ${ }^{9}$.

\section{Antigen uptake by APCs}

Different types of APCs use specific as well as nonspecific mechanisms for antigen uptake ${ }^{10}$. The specific 

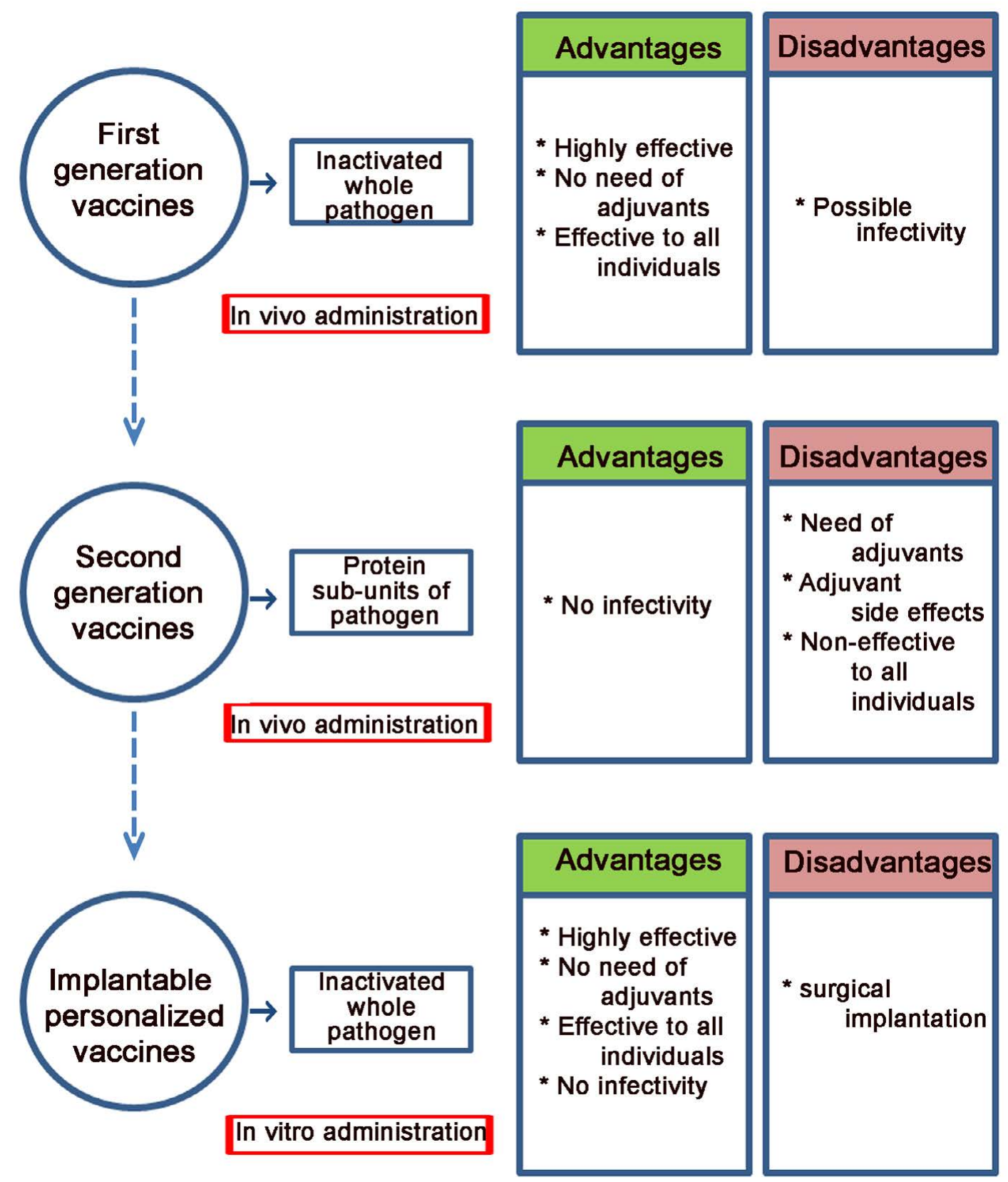

Figure 1: Advantages and disadvantages of first generation vaccine, second generation vaccines and implantable personalized vaccines.

mechanisms are mediated by receptor endocytosis and include immunoglobulin (Ig) molecules on antigen-specific $B$ cells, different types of Fc receptors, mannose receptors for efficient capture of antigen-antibody complexes and mannosylated or fucosylated antigens, C-type lectins (like DEC205) etc. Such specific mechanisms of antigen uptake are accompanied by various signal transduction pathways, leading to the stimulation or suppression of immune activation.

The non-specific mechanisms of antigen uptake include macropinocytosis, which is a cytoskeleton-dependent type of endocytosis, phagocytosis, which is the most important mechanism for foreign antigen uptake by macrophages and some dendritic cells, as well as autophagy, through which cytosolic antigens can be delivered to the antigen processing compartments ${ }^{11}$. Depending on the presence of specific receptors, antigen uptake can lead to different types of cell activation.

Upon uptake the antigen enters the endosomal/ lysosomal compartments, where depending on its nature and concentration, it will be either fully degraded and excreted from the cell or follow the antigen presentation process $^{12,13}$. The nature of the antigen is very important as to the type of immune response development. Thus, 
antigen immunogenicity depends on its foreignness (nonself antigens commonly induce an immune response), the molecular weight (antigens $>14 \mathrm{kD}$ are immunogenic, antigens $<5-10 \mathrm{kD}$ are poorly immunogenic), the chemical nature and heterogeneity (proteins and polysaccharides are mainly immunogenic, heteropolymers are more immunogenic than homopolymers), its physical form (particulate are more immunogenic than soluble antigens), as well as its susceptibility to processing and presentation.

\section{Antigen loading to MHC molecules}

Following the antigen presentation process, partially degraded antigens (antigenic epitopes) will be loaded either to class I or class II MHC molecules to trigger cellular or humoral immunity, respectively. If a substance cannot go through antigen processing, which involves enzymatic digestion creating antigenic epitopes for loading to MHC molecules, then it is a poor immunogen, driving the immune response towards tolerance instead of immunity.

Antigens up taken from the extracellular environment and located within the endosomal compartments are partially degraded by a series of enzymes and will be loaded to class II MHC molecules. The class II presentation pathway has been thoroughly studied ${ }^{14}$. Once the complex MHC class II/antigen is transferred to the cell membrane, it can be recognized by $\mathrm{CD} 4^{+} \mathrm{T}_{\mathrm{H}}$ cells for further stimulation of the immune system.

Intracellular antigens will mainly go through proteosomal degradation, will be driven to ER and delivered to class I MHC molecules ${ }^{15}$. This complex will be recognized by $\mathrm{CD}^{+} \mathrm{T}_{\mathrm{C}}$ cells, initiating the killing process.

These two pathways, as expected in nature, are not mutually exclusive, and different interconnections can take place. Thus, cross-presentation has been described as the process where extracellular antigens are transferred for presentation by class I MHC molecules to $\mathrm{CD}^{+} \mathrm{T}_{\mathrm{C}}$ cells ${ }^{16}$. Similarly, autophagy provides a mechanism by which cells can transfer endogenous (intracellular) antigens into the class II pathway for presentation to $\mathrm{CD}^{+} \mathrm{T}_{\mathrm{H}}$ cells ${ }^{17}$. In addition to this canonical antigen presentation pathway, trogocytosis has been described as the process, where nonAPC cells that fail or do not choose to induce MHC class II molecules by inflammatory mediators can acquire these molecules from neighboring cells through cell-cell contact or by APC-derived exosomes, generating thus MHC-dressed cells ready to trigger T cells ${ }^{18}$.

\section{Immune system manipulation technologies}

Understanding that antigen presentation is a crucial start point for specific immune responsiveness, many researchers of vaccine technology focused their efforts on the development of smart antigen delivery and APC-activation systems. Over the years a number of nanomaterial-based adjuvants have been described to be effective for usage in a variety of antigens, yet safety and perspective mode of action as to the immunostimulatory mechanisms need to be addressed.

The nanomaterial-based antigen delivery systems include the use of nanoparticles and engineered nanomaterials, comprising metals and metal-oxides (aluminium-based, gold and silver nanoparticles, 3D-mesoporous silicon rods), polymers [poly(lacticco-glycolic acid)-PLGA, poly( $\gamma$-glutamic acid)- $\gamma$-PGA, chitosan, polyethyleneimine, $\mathrm{pH}$-responsive polymers) and liposomes ${ }^{19-24}$.

The proposed implantable microneedles, which have been designed to encapsulate the antigen (in most cases influenza virus was applied) and allow slow antigen release and immune activation upon intradermal injection, have been fabricated using a variety of biocompatible polymers ${ }^{25,26}$. Although important advantages have been described, further studies are required to evaluate the response to biomaterial used, the mode of application, the stability of the product and the long-term safety of the procedure ${ }^{27}$.

Mesoporous silica rods have also been proposed for controlled drug release ${ }^{28-30}$. Their effectiveness in recruiting dendritic cells has been shown to be enhanced by the presence of granulocyte- macrophage colony stimulating factor (GM-CSF) ${ }^{21}$. Although mesoporous silica rods might be used as multifunctional platforms in vaccine technology for antigen delivery and immune system manipulation, further studies need to evaluate safety and long lasting efficacy.

Rapid development of material sciences provide a large number of clever solutions for antigen delivery and immune stimulation, yet profound research need to be done by biologists to definitely demonstrate effectiveness, stability, repetitiveness and absence of side effects.

\section{Ex vivo antigen presentation technologies}

Lately, a novel strategy of vaccine technology has been developed, allowing immunization with the inactivated pathogen (known from the first generation vaccines to be the most effective approach), while ensuring safety and no need of adjuvants (Figure 1). This strategy, which lies on the so-called "personalized implantable vaccines", manipulates ex vivo the very first steps of immunization and includes antigen presentation by the host's APCs, loaded (immobilized by natural adherence) onto microtextured Si scaffolds, under controlled culture conditions. Such manipulation resembles the manufacture of an organ-on-chip, since upon subcutaneous implantation, the cell activated Si-scaffold is being merely connected to blood circulation, recruits immune cells and leads to the development of antigen specific memory cells ${ }^{31,32}$. This technology was originally developed using a classic 
protein antigen (human serum albumin) and was later applied to Salmonella Typhimurium and M. luteus-derived peptidoglycan. The proposed strategy of vaccines is meant to be successful to any kind of antigenic stimulus. The need of personalized manipulation might be considered as a problem to the massive needs of immunization in pandemic situations, but in the absence of safe and effective conventional vaccines, these can be easily applied.

A remarkable observation in this strategy of vaccination was that the antigenic load of APCs dictated differential morphology and apparently activity upon subcutaneous implantation, resembling the in vivo generated response to the antigenic stimulus. Under identical experimental procedures, the three antigens described in the literature, namely HSA, S. Typhimurium and peptidoglycan, provided differential scaffold histology, accompanied by specific immune characteristics.

In all cases, laser microstructured Si-scaffolds ${ }^{31,33}$ were loaded with macrophages, which were then activated with the appropriate antigen and implanted to left rear foot of anesthetized mice ${ }^{31,32}$. When the scaffolds were excised seven days later, scanning electron microscopy analysis revealed well distinct scaffold histology among the three antigenic stimuli (Figure 2). In response to HSA, a large number of fibroblasts, adherent macrophages and lymphocytes were observed on the excised scaffold. Lymphocytes were settled on the scaffold-attached macrophages, while highly packed protective membranes, consisting of complex protein depositions and multiple collagen layers surrounding the scaffold, could be observed. Such manipulation was accompanied by high levels of HSAspecific antibody detection in the serum.

In response to $S$. Typhimurium, scaffold removal was an easy abstraction without the need of membranes detachment. In this case, the organism had not made such strong membranes for the isolation of the scaffold. The tissue around the structure was one set of loose membranes (Figure 2B), but this did not discourage the organism to produced specific antibodies, as detected in the serum.

In response to peptidoglycan, scaffold was also removed easily, but in this case only minimal cases of macrophages attached with lymphocytes on their surface could be observed (Figure 2C). In this case, although the manipulation resulted in increased IgG levels in the serum, no antigen specific antibody could be detected. This observation, along with the increased cytokine levels and white cell numbers led to the hypothesis that M. luteus peptidoglycan was likely to act as a mitogen.

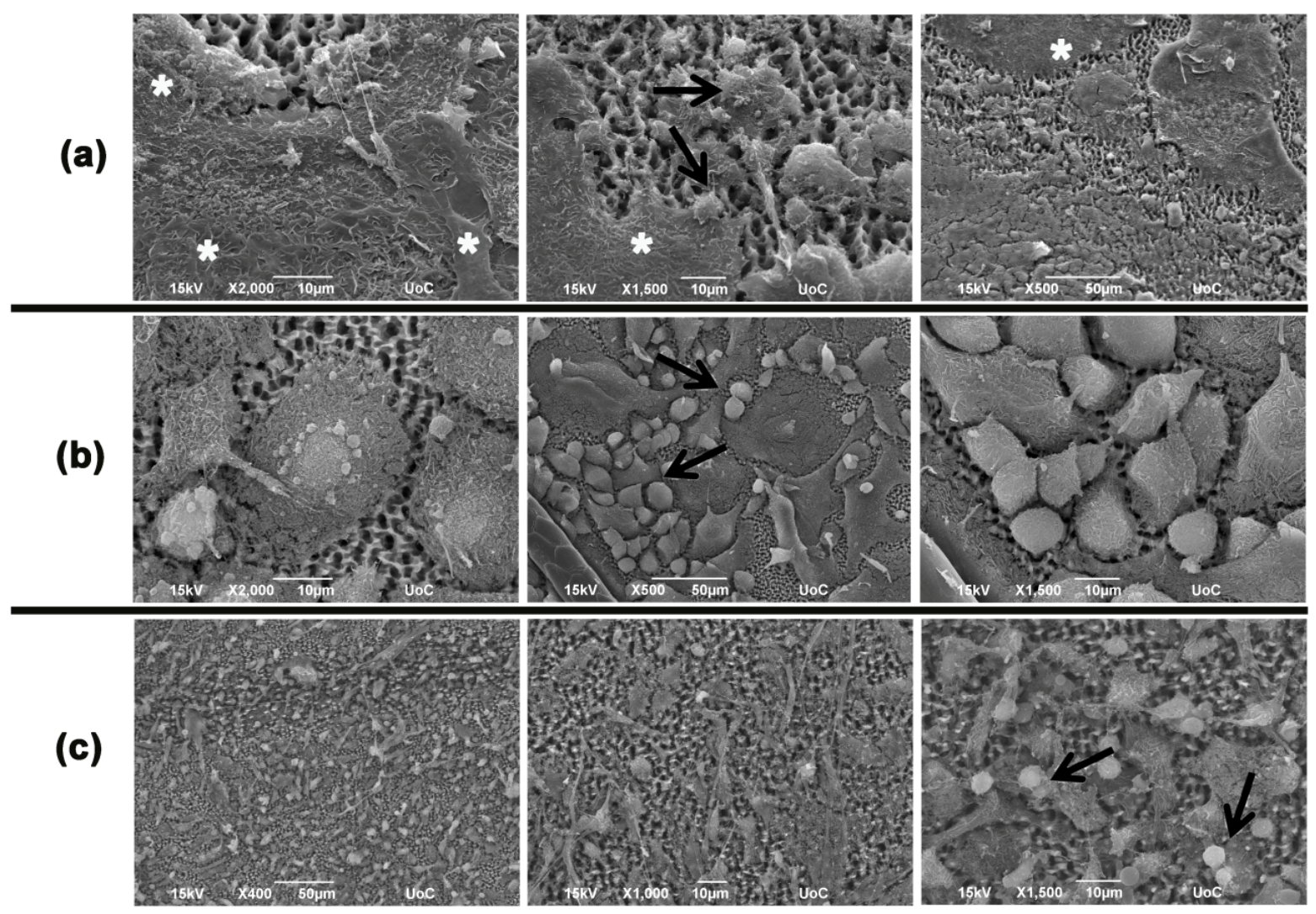

Figure 2: Laser microstructured Si-scaffolds loaded with macrophages and activated by HSA (a), S.typhimurium extracts (b) and M. luteus-derived peptidoglycan (c). Asterisks show some areas of protein depositions and collagen layers. Arrows show some examples of lymphocyte contacts with adherent cells 
These observations show that indeed, the nature of the antigenic stimulus may dictate a whole different response to the organism. Therefore, universal adjuvants or antigendelivery systems cannot be effective for every antigen and each organism. The proposed ex vivo antigen presentation technology allows to finely tune immune response to the required direction. Indeed, one could foresee the use of peptidoglycan as a mild mitogen to be provided along with a weak immunogenic antigen, or provided as a mild immunostimulant against immunosuppressive conditions like sepsis and cancer.

\section{Conclusions}

Although $\mathrm{T}$ cell responses dictate the faith of the immune response, their activation follows a series of events, which are responsible for the nature of $\mathrm{T}$ cell activation process. Depending on how the antigen will be delivered to the organism, the mode by which the antigen will be up-taken by the APCs, the pathway to be followed during the formation of the MHC-antigen complex and its presentation or not to the cell membrane are crucial parameters, allowing or not antigen recognition. Since a great deal of knowledge is still missing, it is extremely difficult to predict the effectiveness of universal practices during immune system manipulation. The ex vivo personalized immunization discussed herein seems to implicate minimal immune manipulation, allowing naturally selected antigenic load, and tunable immune manipulation.

\section{Conflict of interest}

The authors declare no financial or commercial conflicts of interest

\section{References}

1. Burgdorf S, Kurts C. Endocytosis mechanisms and the cell biology of antigen presentation. Curr Opin Immunol, 2008; 20: 89-95.

2. Rock KL, Reits E, Neefjes J. Present yourself by MHC class I and MHC class II molecules.Trends Immunol, 2016; 37: 724-737.

3. Siddiqui S, Visvabharathy L, Wang CR. Role of group 1 CD1-restricted T cells in infectious disease. Front Immunol, 2015; 6: 337. doi: 10.3389/ fimmu.2015.00337.

4. Riedel S. Edward Jenner and the history of smallpox and vaccination. Proceedings (Baylor University. Medical Center), 2005; 18(1): 21-25.

5. Reed SG, Bertholet S, Coler RN, Friede M. Novel perspectives for influenza vaccine formulation and administration. Trends Immunol, 2009; 30: 23-32

6. Aguilar JC, Rodriguez EG. Vaccine adjuvants revisited. Vaccine, 2007 25: 3752-3762.

7. Lindblad EB. Aluminium compounds for use in vaccines. Immunol Cell Biol, 2004; 82: 497-505.

8. He P, Zou Y, Hu Z. Advances in aluminum hydroxide-based adjuvant research and its mechanism. Hum Vaccin Immunother, 2015; 11: 477488.

9. Chen X, Kim P, Farinelli B, Doukas A, Yun SH, Gelfand JA, Anderson $\mathrm{RR}$, Wu MX. A novel laser vaccine adjuvant increases the motility of antigen presenting cells. PloS One, 2010; 5: 1-11.

10. Lanzavecchia A. Mechanisms of antigen uptake for presentation. Cur Opin Immunol, 1996; 8(3): 348-354.

11. Liu Z, Roche PA. Macropinocytosis in phagocytes: regulation of MHC class-II-restricted antigen presentation in dendritic cells. Front Physiol, 2015; 6. doi:10.3389/fphys.2015.00001

12. R M Steinman, I S Mellman, W A Muller, Z A Cohn. Endocytosis and the recycling of plasma membrane. J Cell Biol, 1983; 96(1): 1-27.

13. Mahmutefendić H, Blagojević Zagorac G, Maćešić S, Lučin P. Rapid Endosomal Recycling, in Peripheral Membrane Proteins. Edited by Shihori Tanabe, p. 83-104, 2018.

14. Rocha N and Neefjes J. MHC class II molecules on the move for successful antigen presentation. EMBO J, 2008; 27: 1-5.

15. Hulpke S, Tampe R. The MHC I loading complex: a multitasking machinery in adaptive immunity. Trends Biochem Sci, 2013; 28: 412420.

16. Norbury CC. Defining cross presentation for a wider audience. Cur Opin Immunol, 2016; 40:110-116

17. Münz C. Autophagy beyond intracellular MHC Class II antigen presentation. Trends Immunol, 2016; 37: 755-763.

18. Nakayama M. Antigen Presentation by MHC-Dressed Cells. Front Immunol, 2015; doi: 10.3389/fimmu.2014.00672 1-5.

19. Sun B, Xia T. Nanomaterial-Based Vaccine Adjuvants. J. Mater. Chem. B, 2016; 4: 5496-5509.

20. Chen YH, Lai KY, Chiu YH , Wu YW , Shiau AL, Chen MC. Implantable microneedles with an immune-boosting function for effective intradermal influenza vaccination. Acta Biomaterialia, 2019; 97: 230-238.

21. Kim J, Li WA, Choi Y, Lewin SA, Verbeke CS, Dranoff G, Mooney DJ Injectable, spontaneously assembling, inorganic scaffolds modulate immune cells in vivo and increase vaccine efficacy. Nat Biotech, 2015; 33:64-72.

22. [Moon JJ, Suh H, Bershteyn A, Stephan MT, Liu H, Huang B, Sohail M, Luo S, Um SH, Khant H, Goodwin JT, Ramos J, Chiu W, Irvine DJ. Interbilayer-crosslinked multilamellar vesicles as synthetic vaccines for potent humoral and cellular immune responses. Nat Mater, 2011; 10: 243-251

23. St. John AL, Chan CY, Staats HF, Leong KW, Abraham SN. Synthetic mast-cell granules as adjuvants to promote and polarize immunity in lymph nodes. Nat Mater, 2012; 11: 250-257.

24. Gosselin EA, Eppler H B, Bromberg J S, Jewell CM. (2018). Designing natural and synthetic immune tissues. Nat Mater, 2018; 17(6): 484498.

25. Sullivan S, Koutsonanos D, del Pilar Martin M, Lee JW, Zarnitsyn V, Choi SO, Murthy N, Compans RW, Skountzou I, Prausnitz MR. Dissolving polymer microneedle patches for influenza vaccination. Nat Med, 2010; 16, 915-920. (2010).

26. Hirobe S, Azukizawa H, Hanafusa T, Matsuo K, Quan YS, Kamiyama F, Katayama I, Okada N, Nakagawa S. Clinical study and stability assessment of a novel transcutaneous influenza vaccination using a dissolving microneedle patch. Biomaterials, 2015; 57: 50-58.

27. Leone M, Mönkäre J, Bouwstra JA, Kersten G. Dissolving Microneedle Patches for Dermal Vaccination. Pharm Res, 2017; 34: 2223-2240.

28. Xia T, Kovochich M, Liong M, Meng H, Kabehie S, George S, Zink JI, Nel AE. Polyethyleneimine coating enhances the cellular uptake of mesoporous silica nanoparticles and allows safe delivery of siRNA and DNA constructs. ACS Nano, 2009; 3: 3273-3286.

29. Kim J, Kim HS, Lee N, Kim T, Kim H, Yu T, Song IC, Moon WK, Hyeon T. Multifunctional uniform nanoparticles composed of a magnetite 
nanocrystal core and a mesoporous silica shell for magnetic resonance and fluorescence imaging and for drug delivery. Angew Chem Int Ed Engl, 2008; 47: 8438-8441.

30. Li, Z., Barnes, J.C., Bosoy, A., Stoddart, J.F. \& Zink, J.I. Mesoporous silica nanoparticles in biomedical applications. Chem Soc Rev, 2012; 41; 2590-2605.

31. Zerva I, Simitzi C, Siakouli-Galanopoulou A, Ranella A, Stratakis E, Fotakis C, Athanassakis I. Implantable vaccines: In vitro antigen presentation enables in vivo immune response. Vaccine, 2015; 33: 3142-3149.
32. Zerva I, Katsoni E, Simitzi C, Stratakis E, Athanassakis I. Laser microstructured Si scaffold implantable vaccines against Salmonella Typhimurium. Vaccine, 2019; 37: 2249-2257. doi.org/10.1016/j. vaccine.2019.02.080

33. Simitzi C, Stratakis E, Fotakis C, Athanassakis I, Ranella A. Microconical silicon structures influence NGF induced PC12 cell morphology. J Tissue Eng Regen Med, 2014; DOI: 10.1002/term.1853. 